\title{
Adsorption of a Cholesteric Liquid Crystal Polyester on Silver Nanoparticles Studied by Surface-Enhanced Raman Scattering and Micro Raman Spectroscopy
}

\author{
M. PÉREZ-MÉNDEZ, R. MARSAL-BERENGUEL, and S. SANCHEZ-CORTES* \\ Instituto de Ciencia y Tecnología de Polímeros, CSIC, Juan de la Cierva, 3. 28006 Madrid (M.P.-M., R.M.-B.); and Instituto de \\ Estructura de la Materia, CSIC, Serrano, 121. 28006 Madrid (S.S.-C.)
}

\begin{abstract}
A Raman study of the adsorption of thermotropic cholesteric liquid crystal polyester PTOBDME $\left(\left[\mathrm{C}_{34} \mathrm{H}_{36} \mathrm{O}_{8}\right]_{n}\right)$ on Ag surfaces is presented in this work. The affinity and adsorption mechanism of this polymer was tested on Ag metal colloids and on Ag films obtained by direct immobilization of the colloidal nanoparticles. We have first studied the structure of PTOBDME suspended in several solvents in order to identify the Raman bands used as structural markers. The adsorption of the polymer leads to a deep conformational change involving both the main chain and the aliphatic side chain. The interaction of polymers like PTOBDME with metals could be interesting in the formation of functionalized surfaces, providing them with specific physicohemical properties with possible applications in recognition phenomena, which can be easily characterized by Raman spectroscopy.
\end{abstract}

Index Headings: Cholesteric liquid crystal; Surface-enhanced Raman scattering; SERS; Environmental scanning electron microscopy; ESEM; Adsorption; Silver nanoparticles.

\section{INTRODUCTION}

Liquid crystals are self-organizing systems that combine the order of perfect crystals with the mobility of gases and liquids. Liquid crystal molecules have anisotropic or amphiphilic shape, combining a rigid geometry (mesogen units) with a flexible spacer, building blocks with anisotropic properties. ${ }^{1}$

They do not go directly from the crystalline state into the isotropic state. Instead, they form intermediate mesophases. In the mesophase state, they orient with their longitudinal molecular axes parallel. Two major classes of mesophases can be distinguished: nematic (with molecular centers organized isotropically), and smectic (with molecular centers organized in layers). The special array of nematic planes stacked in a "superhelical" three-dimensional structure, with a prevalent screw direction, is called the cholesteric mesophase. Cholesteric mesophases reflect selectively incident light and when the length of the helical pitch is comparable to the wavelength of visible light, they exhibit typically brilliant colors. ${ }^{2}$

Thermotropic cholesteric liquid crystal polyesters have received much attention in the last few years as engineering polymers for specific applications. These materials have interesting mechanical and optical properties, which has allowed their application in many engineering fields, such as photonics, due to the special optical properties of these materials. ${ }^{3}$

We have previously reported the synthesis of thermo-

Received 11 September 2003; accepted 13 January 2004.

* Author to whom correspondence should be sent. E-mail: imts158@ iem.cfmac.csic.es. tropic liquid crystal: Poly[oxy(1,2-dodecane)oxycarbonyl1,4-phenyleneoxycarbonyl-1,4-phenylenecarbonyloxy1,4-phenylenecarbonyl], PTOBDME, $\left[\mathrm{C}_{34} \mathrm{H}_{36} \mathrm{O}_{8}\right]_{\text {n }}$ (Fig. 1), obtained as cholesteric, chiral in nature, although synthesized from the racemic mixture of DL-1,2-dodecanediol. ${ }^{4}$

Within the amphiphilic monomer molecule of PTOBDME, two parts can be distinguished: the mesogene, rigid moiety: terephthaloyl-4-bis-oxybenzoate (TOB), along the main chain, containing the polar groups (two terephthalate carbonyl groups, two oxybenzoate carbonyl groups, and three benzene rings); and a flexible spacer along the lateral chain, with hydrophobic, aliphatic structure (Fig. 1). The polymerization of such monomer molecules always displays a helical structure with a spiral staircase conformation. We have reported its conformational analysis by molecular mechanics. ${ }^{5}$ Benzene rings are torsioned through the ester groups, avoiding coplanarity, and twisting along the main chain, giving rise to helical "cages", while the aliphatic residues are directed to the inner part of the helix ${ }^{5}$ (Fig. 2).

The self-assembling of PTOBDME in helical cavities, heterogeneous in size and between 450 and $200 \mathrm{~nm}$, when dispersed in aqueous solution has been experimentally observed by fluorescence and Raman spectroscopy. The conformation of these cavities changes depending on the solvent in which the polymer is suspended. This property enables these materials to entrap smaller molecules inside, such as drugs and biomolecules, with potential application as carriers. ${ }^{6}$

Also, we have proved, by steady-state fluorescence, that PTOBDME interacts with liposomes of any chemical composition and effective charge, either by inserting their aliphatic lateral chains or the whole helical molecules.

Raman spectroscopy has been successfully applied in the study of this type of polymer. We have used this technique for the structural characterization of PTOBDME, providing useful structural information on both the lateral side chain and the mesogenic moieties. ${ }^{5}$

The adsorption of this polymer on a metal surface could be of interest due to the combination of the properties of both the polymer and the metal. In fact, the adsorption of liquid crystal polyester on surfaces has been investigated in the preparation of functionalized surfaces with tailor-made structures that can act as new materials with special properties regarding processes of marked industrial or biological interest such as sensors, optoelectronic devices, or molecular recognition or simply as water repelents. ${ }^{7}$ In the study of liquid crystal adsorption on a surface, one must take into account that the 


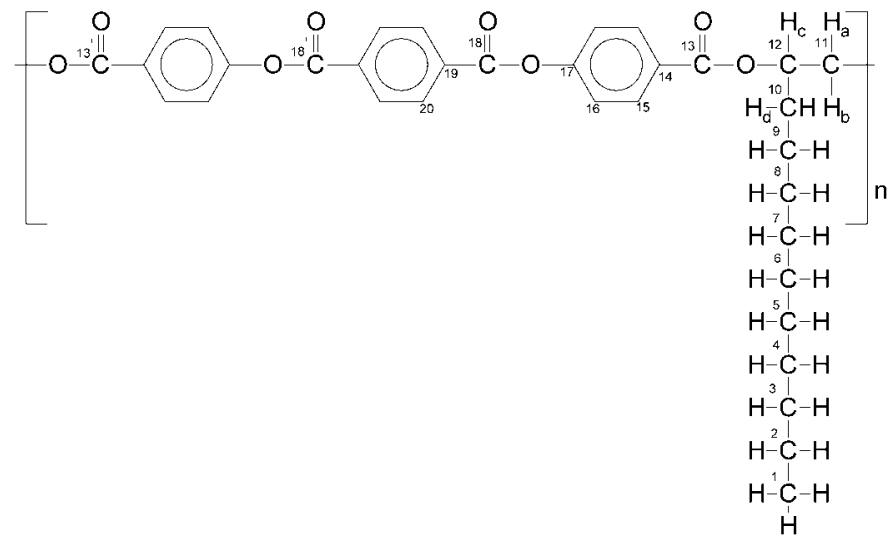

FIg. 1. Formula of PTOBDME.

interaction of the polymer largely depends on its orientation and structure, ${ }^{8}$ and this is very important in controling the physicochemical polymer properties, as well as the ulterior applications of the obtained devices. In this work we report the study of the adsorption capabilities of PTOBDME on several metal surfaces.

In order to study the adsorption of PTOBDME on metal surfaces we have used surface-enhanced Raman scattering (SERS) spectroscopy. This technique is nowadays an active area with a broad range of analytical applications, which can be successfully applied in the study of interfacial phenomena. ${ }^{9}$ Since its discovery, ${ }^{10}$ SERS has become a common technique for analyzing the adsorption of molecules on surfaces. Although this technique is based on the enormous enhancement of the electromagnetic field occurring in the vicinity of metallic nanoparticles, mainly $\mathrm{Ag}$ and $\mathrm{Au},{ }^{11}$ a chemical contribution has also been suggested to explain the overall SERS enhancement related to a charge transfer between the adsorbate and the metal surface. ${ }^{12}$ The short-range enhancement occurring in SERS together with the surface selection rules, ${ }^{13,11 a}$ which predict a more intense enhancement for those vibrational modes closer and perpendicular to the metal, can be used to investigate the electrodynamic behavior of organic molecules adsorbed on charged surfaces, thus providing very valuable information regarding the structure of the adsorbed molecules.

A micro-Raman probe allows the study of very small volumes and surfaces. The combination of micro-Raman and SERS techniques (micro-SERS) together with the use of highly sensitive detection systems leads to a remarkable increase of the sensitivity of Raman experiments. This combination of experimental devices could be useful in the study of the adsorption of large molecules whose adsorption mechanism could locally change.

In the present work, the normal Raman, SERS, and micro-SERS techniques were applied in the study of the conformation of PTOBDME at different conditions and its interaction and orientation on $\mathrm{Ag}$ surfaces prepared by different procedures at different excitation wavelengths. This study aimed to (1) investigate the self-assemblying properties of PTOBDME; (2) check the best metal surface for the adsorption of this polymer in oder to prepare functionalizated metal surfaces acting as special devices in recognition phenomena; and (3) implement a characterization method of the prepared metal surfaces based

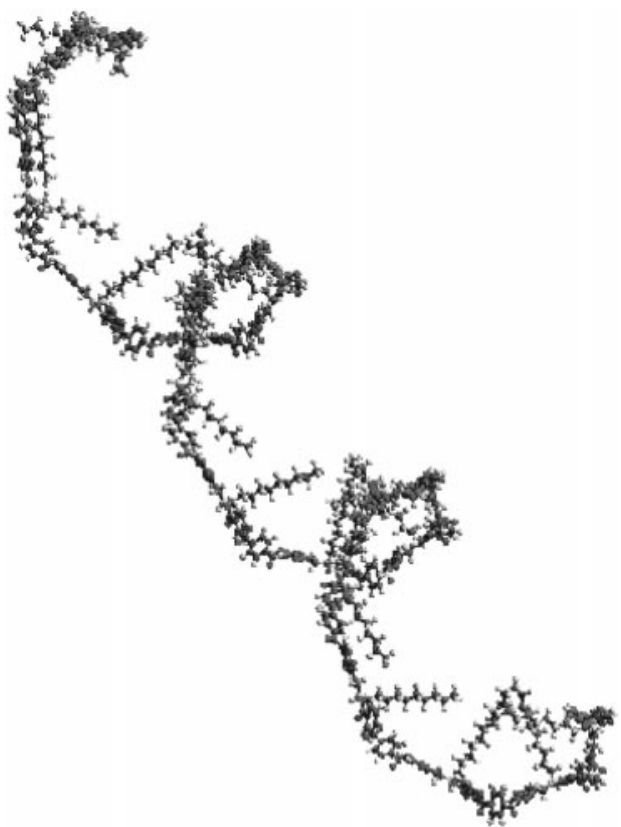

FIG. 2. (PTOBDME) $)_{20}$ helical model.

on the Raman spectroscopy and microscopies of different nature.

\section{EXPERIMENTAL}

Materials. PTOBDME was synthetized as previously reported. ${ }^{4}$ All reagents were of analytical grade.

Preparation of Metal Colloids. Silver colloids for SERS measurements were prepared by reducing $\mathrm{AgNO}_{3}$ with citrate. ${ }^{14}$ Triply distilled water was employed in the preparation of all the solutions.

The Ag colloids prepared in this way displayed extinction bands centered at $430 \mathrm{~nm}$. In previous works we accomplished a detailed optical and microscopic study of citrate colloids. ${ }^{15,16}$

Sample Preparation for Normal Raman. Samples in solution for normal Fourier transform (FT) Raman experiments were obtained by suspending $2 \mathrm{mg}$ of the polymer in $500 \mu \mathrm{L}$ of the corresponding solvent. The suspension was placed in $0.5-\mathrm{cm}$ optical pathway quartz cuvettes. For solid samples, the powder was placed inside a brass holder to record the FT-Raman spectra or was directly deposited on a glass slide to record the Raman spectrum at $785 \mathrm{~nm}$.

Samples Preparation for Surface-Enhanced Raman Scattering. Samples in solution for SERS measurements were obtained by suspending $2 \mathrm{mg}$ of the polymer in 500 $\mu \mathrm{L}$ of ethanol. Then a $2 \mu \mathrm{L}$ aliquot of this suspension was added to $250 \mu \mathrm{L}$ of the Ag colloid. The Ag colloid was then aggregated to achieve a higher SERS intensity by adding aliquots of an aqueous potassium nitrate solution $\left(5 \times 10^{-1} \mathrm{M}\right)$ up to a $10 \mathrm{mM}$ final concentration. Aliquots of 5 to $6 \mu \mathrm{L}$ were put in 2-mm-diameter glass capillaries to obtain the SERS spectra at different excitation wavelengths.

Samples for micro-SERS measurements in the immobilized Ag nanoparticle films were prepared by depositing an aliquot $(20 \mu \mathrm{L})$ of the suspension described in the previous paragraph onto a glass slide and then allowing 
the solvent to evaporate at room temperature. Afterwards, the so-obtained film was washed with water and ethanol in order to remove any salts or citrate (or its oxidation products) remaining in the sample. Finally, a drop of water was deposited onto the obtained film and, after, a glass cover slide was put on the drop. This experimental setup avoided the possible photothermal decomposition of the sample induced by the laser irradiation.

Instrumentation. Normal Raman spectra were recorded in a U-1000 Jobin-Yvon spectrophotometer by exciting with the $514.5 \mathrm{~nm}$ radiation line of a Spectra Physics Model 165 argon ion laser equipped with a thermoelectrically cooled Products for Research, Inc. photon counting system photomultiplyer tube. The resolution was set at $4 \mathrm{~cm}^{-1}$ and a $90^{\circ}$ geometry was used to record the data. The laser power on the sample was fixed at $30 \mathrm{~mW}$. The spectra reported in this work were recorded at $1 \mathrm{~cm}^{-1}$ step intervals with an integration time of $1 \mathrm{~s}$. Four to five scans were averaged to obtain the SERS spectra shown in this work.

The Raman spectra obtained with the $785 \mathrm{~nm}$ line were obtained in a Renishaw Raman Microscope System RM1000 equipped with a Leica microscope and a chargecoupled device (CCD) camera. The spectra shown here were obtained by using an NPLAN $100 \times$ objective. A laser power of $1 \mathrm{~mW}$ at the sample was employed. One scan of $10 \mathrm{~s}$ was collected each time.

Fourier transform-Raman spectra were obtained by using an RFS 100/S Brucker spectrophotometer equipped with a Ge detector cooled by liquid nitrogen. The 1064 $\mathrm{nm}$ line, provided by a $\mathrm{Nd}$ : YAG laser, was used as the excitation line. Resolution was set to $4 \mathrm{~cm}^{-1}$ and a $180^{\circ}$ geometry was employed. The laser power at the sample was $150 \mathrm{~mW}$. The FT-SERS spectra shown here are the result of accumulating 1000 scans during $20 \mathrm{~min}$. The resulting FT-Raman spectra are shown without smoothing.

A PHILIPS XL30 Environmental Scanning Electron Microscope (ESEM) was used to analyze the morphology of our samples. These did not need to be covered by any conducting material, as they could be studied under environmental conditions. An electronic servopressure system kept the sample holder chamber in a range between 1-20 torr (within an accuracy of 0.1 torr). Microphotos were attained at $2{ }^{\circ} \mathrm{C}$. Samples for environmental scanning electron microscopy (ESEM) were obtained by depositing the sample solution on a steel support for SEM microscopy, and then the solvent was allowed to evaporate.

\section{RESULTS AND DISCUSSION}

Raman Spectra of PTOBDME in Solid and Suspended in Different Solvents. Before analyzing the adsorption of PTOBDME on metals we carried out a conformational study of the polymer in different solvents by Raman spectroscopy.

In the three panels of Fig. 3 we show the Raman spectra of PTOBDME in both the solid state (a) and suspended in different solvents: aqueous solution (b), chloroform $\left(\mathrm{CHCl}_{3}\right)$ (c), and dimethyl sulfoxide (DMSO) (d). The spectra are mainly dominated by the contribution of the mesogens (TOB) (terephthaloyl-4-bis-oxybenzoate),

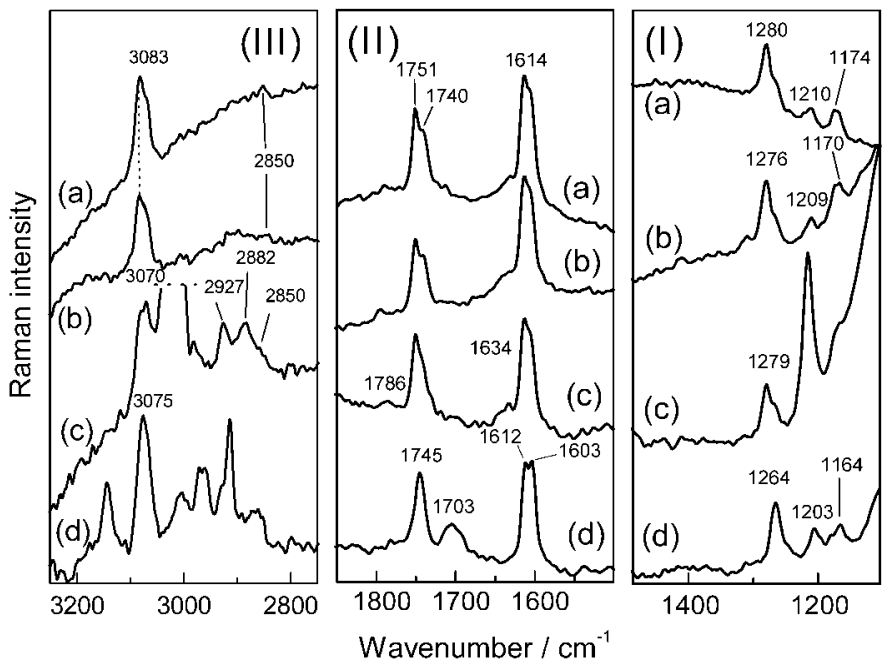

FIG. 3. FT-Raman spectra of PTOBDME in: (a) solid state; (b) water suspension; (c) $\mathrm{CHCl}_{3}$; and (d) DMSO. Excitation: $1064 \mathrm{~nm}$.

rigid parts of the molecule, along the polymer main chain, while the bands corresponding to the aliphatic lateral chains with different vibrational modes (see Fig. 1) are very weak.

The most intense bands of the mesogen moiety correspond to the ester and aromatic double bond stretching, which appear above $1600 \mathrm{~cm}^{-1}$. These bands undergo significant changes when varying the solvent where the polymer is suspended.

The Raman spectrum of PTOBDME suspended in $\mathrm{CHCl}_{3}$ (Fig. 3II, spectrum c) shows intense bands at 1751 and $1614 \mathrm{~cm}^{-1}$ that are attributed to $\mathrm{C}=\mathrm{O}$ stretching motions of ester carbonyls and $\mathrm{C}=\mathrm{C}$ stretching motions of the aromatic rings. These bands are relatively shifted upwards in relation to polyethylene terephthalates, ${ }^{17}$ and in relation to other lineal polymers containing TOB as the mesogen group. ${ }^{18}$ This effect is attributed to the different structure adopted by the TOB moiety in the PTOBDME solvated in $\mathrm{CHCl}_{3}$. In fact, as mentioned above, this polymer displays a helicoidal structure where the TOB rings are rotated, while the aliphatic residues are directed to the inner part of the helix (Fig. 2). This special structure leads to a large torsion in the benzene ring planes through the ester groups, avoiding the co-planarity between aromatic rings and the connecting ester planes. As a consequence of this structure, the electronic resonance between aromatic rings is markedly reduced, and the double bond character of $\mathrm{C}=\mathrm{O}$ and $\mathrm{C}=\mathrm{C}$ bonds is increased. This is the reason for observing a frequency shift upwards.

The existence of a shoulder at $1604 \mathrm{~cm}^{-1}$ in $\mathrm{CHCl}_{3}$ suggests that the polymer could also adopt a different structure in some parts of the chain. In the solid state (Fig. 3II, spectrum a) and in aqueous suspension (Fig. 3II, spectrum b) the shoulders at 1740 and $1604 \mathrm{~cm}^{-1}$ are markedly intensified, suggesting a more pronounced structural change of the polymer in terms of a higher planarity between the ester group and benzene rings in the latter media. In fact, in more planar polymers bearing a lineal structure, integrated by polymethylene chains linked to TOB sequences, these bands appear at a lower frequency due to the lower torsion of TOB triad planes 
in this case, ${ }^{18}$ as corresponds to a higher electronic resonance at higher planarity.

The positions of the $v(\mathrm{C}=\mathrm{O})$ and $v(\mathrm{C}=\mathrm{C})$ stretching bands can thus be considered as markers for the planarity between rings in polymers of this kind, and hence, is a valuable estimation of the helical pitch in those chiral polymers. According to the results shown above, we can conclude that PTOBDME adopts a more coiled helical structure in $\mathrm{CHCl}_{3}$ than in solid or in water suspension, where the polymer seems to be partially opened, adopting a more extended conformation. These conformational changes could not be general due to the remaining presence of $v(\mathrm{C}=\mathrm{O})$ and $\nu(\mathrm{C}=\mathrm{C})$ bands corresponding to the coiled polymer in water. In fact, the presence of the bands at 1750 and $1740 \mathrm{~cm}^{-1}$ indicates the coexistence of two different structures, corresponding to coiled and uncoiled PTOBDME chains. This is also the case for the suspension in DMSO, where the bands at 1745 and $1603 \mathrm{~cm}^{-1}$ indicate the existence of a mixture of coiled and uncoiled polymer structures.

Bands due to double bonds are not uniquely affected by a change in the torsion angles between planes in TOB moieties. For instance, the bands appearing at 1280/1260 and $1210 \mathrm{~cm}^{-1}$ (Fig. 3I), which are attributed to $v(\mathrm{C}-\mathrm{O}-$ C) stretching vibrations coupled to $\delta(\mathrm{CH})$ vibrations of aromatic rings, ${ }^{19}$ also undergo a change in frequency when the polymer is placed in different solvents. These bands tend to shift downwards in $\mathrm{CHCl}_{3}$ and in DMSO (the shoulder at $1264 \mathrm{~cm}^{-1}$ is more prominent in $\mathrm{CHCl}_{3}$ and DMSO, while the second band is shifted to 1203 $\mathrm{cm}^{-1}$ in DMSO). These results agree well with the assumption that the polymer helix formed in these solvents is more coiled, leading to a lower electronic resonance between rings and a lower planarity between rings. In the case of DMSO there is a certain mismatch between the behavior of the $v(\mathrm{C}=\mathrm{O})$ and $v(\mathrm{C}=\mathrm{C})$ bands and that of the $v(\mathrm{C}-\mathrm{O}-\mathrm{C})$ stretching vibrations, which we attribute to the possible intermolecular interaction of PTOBDME chains in this solvent, like other related polymers in suspension, ${ }^{20}$ or to a specific interaction of the solvent with the polymer helical structure. Therefore, solvent polarity has a great influence on the PTOBDME structure: in the nonpolar solvent $\mathrm{CHCl}_{3}$, the polymer tends to adopt a preferential helical structure, where the solvent molecules could also be included inside the helical structure participating in its stabilization, while in polar solvents (DMSO and water) the helical structure seems to be partially broken, leading to the coexistence of coiled and uncoiled polymer chains.

The conformational change of PTOBDME is also manifested in the high wavenumber region (Fig. 3III). In this region, very weak bands due to aliphatic $\mathrm{C}-\mathrm{H}$ stretching between 3000 and $2800 \mathrm{~cm}^{-1}$ can be observed, although there is a long polymethylene chain. We attribute this to the fact that the side chains are placed inside the polymer helicoidal structure. This is probably the reason why the $\mathrm{CH}_{2}$ deformation, usually appearing at $1450 \mathrm{~cm}^{-1}$, is very weak in the Raman spectrum (see Fig. 3I). In contrast, the $\mathrm{C}-\mathrm{H}$ stretching vibration in aromatic groups, appearing at $3083-70 \mathrm{~cm}^{-1}$, is very strong and seems to also be sensitive to the PTOBDME structure. The position of this band depends on the electronic delocalization in aromatic rings. In all the cases we have observed a doublet

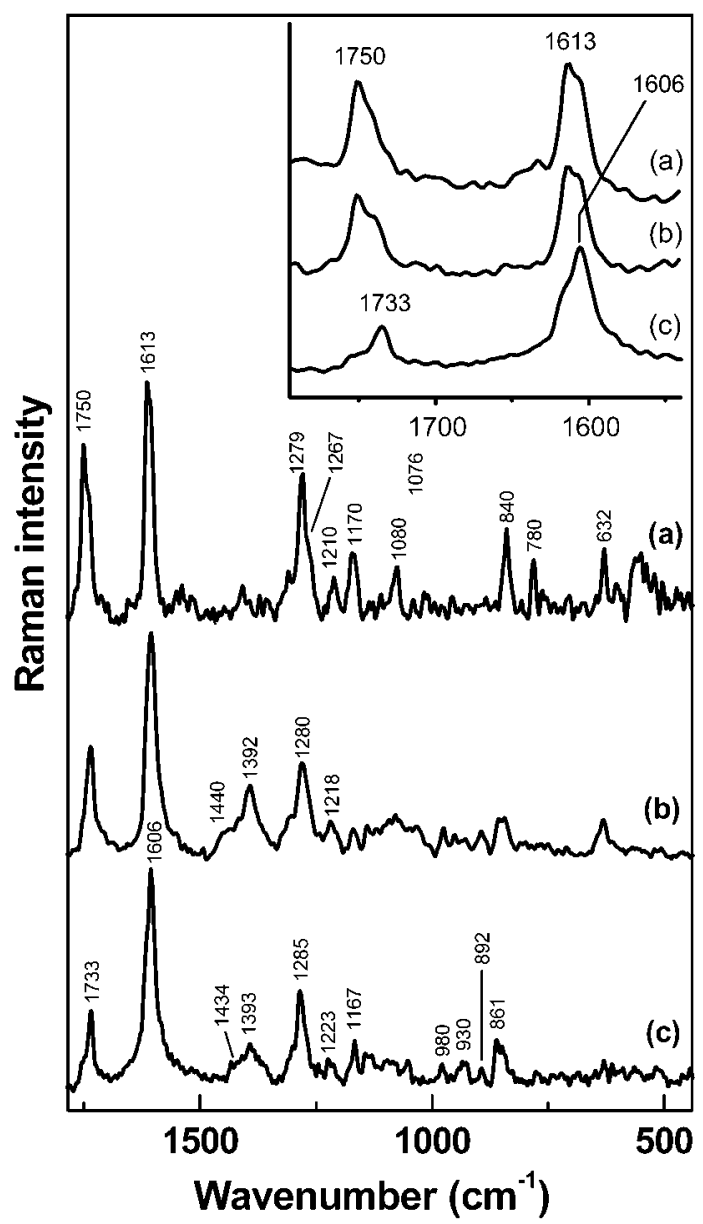

FIG. 4. Raman of PTOBDME in (a) water suspension and SERS spectra of the polymer on Ag citrate colloid by exciting at $(\boldsymbol{b}) 1064 \mathrm{~nm}$ and (c) $514.5 \mathrm{~nm}$. Inset: Raman spectra of PTOBDME in (a) $\mathrm{CHCl}_{3},(\boldsymbol{b})$ water, and SERS at $(\boldsymbol{c}) 514.5 \mathrm{~nm}$ showing in detail the $\mathrm{C}=\mathrm{O}$ and $\mathrm{C}=\mathrm{C}$ stretching region.

at the position of $\mathrm{C}-\mathrm{H}$ stretching appearing at 3083 and $3070 \mathrm{~cm}^{-1}$. The highest component predominates in aqueous medium while the lowest one is more intense in $\mathrm{CHCl}_{3}$. This result can be related again to the lower planarity of aromatic rings as corresponds to a higher torsion of the mesogens along the main chain in the last solvent.

Although the aliphatic $\mathrm{C}-\mathrm{H}$ stretching bands appearing below $3000 \mathrm{~cm}^{-1}$ are very weak, the structural changes undergone by PTOBDME at the different conditions are reflected in this region as well. Usually, the $I_{2890} / I_{2850}$ ratio has been employed as a marker of the lateral packing between aliphatic chains in phospholipids and related compounds. ${ }^{21}$ This ratio undergoes an increase in going from water to chloroform, indicating that in the last solvent the aliphatic side chains are more ordered, and this is probably associated with the helicoidal structure adopted by PTOBDME with a lower planarity between aromatic planes.

Surface-Enhanced Raman Spectra of PTOBDME in Ag Colloids. The SERS spectra of PTOBDME on a citrate Ag colloid are shown in Fig. 4 at different excitation wavelengths. The spectra recorded at 1064 and $514.5 \mathrm{~nm}$ (Figs. 4b and 4c, respectively) are very similar, although there are slight differences between them. When compared to the Raman spectrum of PTOBDME in water 


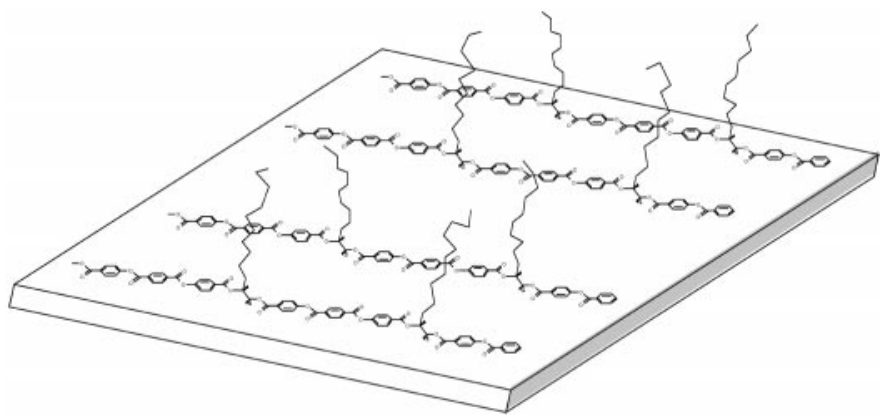

FIG. 5. Adsorption of extended PTOBDME on the metal surface.

(Fig. 4a), the SERS bands corresponding to $\mathrm{C}=\mathrm{O}$ and $\mathrm{C}=\mathrm{C}$ stretching motions undergo a shift downwards to 1733 and $1605 \mathrm{~cm}^{-1}$, respectively. This means a shift of 18 and $8 \mathrm{~cm}^{-1}$, respectively. Besides, the $\mathrm{C}=\mathrm{O}$ band undergoes a marked intensity decrease in relation to the water suspension. In a recent work by McAnally et al., a similar spectral result was found for poly(ethylene terephthalate) adsorbed on Ag nanoparticles studied by SERS. ${ }^{17 \mathrm{c}}$ In the right-hand upper corner inset figure, we show in more detail the region corresponding to these bands. Note that these wavenumber values are close to those observed for lineal TOB polymers, where the torsion between ring and ester groups is supposed to be much lower. Since the latter bands are related to a conformational change of PTOBDME, their marked downward shift suggests that the polymer is more extended when adsorbed onto the metal surface, the torsional angle between benzene rings being smaller. We suggest that PTOBDME is adsorbed on the metal in an uncoiled structure in such a way that the TOB benzene ring planes may be only slightly rotated along the polymer main chain. This structural change may be favored by an interaction of the metal with the aromatic rings via $\pi$-electronic interactions. This mechanism induces the polymer uncoiling in order to ensure a similar approach of rings to the metal surface while the aliphatic side chain is directed towards the bulk solution according to the scheme depicted in Fig. 5.

The higher planarity existing in the TOB moiety triad and the resonance increase between rings and ester moieties leads to a general upwards shift of the bands at $1279 / 1267$ and $1210 \mathrm{~cm}^{-1}$ towards $1285 \mathrm{~cm}^{-1}$ (with the disappearance of the shoulder at $1267 \mathrm{~cm}^{-1}$ ) and 1223 $\mathrm{cm}^{-1}$ (in the SERS registered at $514.5 \mathrm{~nm}$ ), respectively.

In the $1500-1350 \mathrm{~cm}^{-1}$ region we have observed the enhancement of a band at about $1392 \mathrm{~cm}^{-1}$ and of a shoulder at $1430 / 40 \mathrm{~cm}^{-1}$, which are attributed to the aliphatic part of the polymer that is placed closer to the metal surface as a consequence of the polymer uncoiling, e.g., it must correspond to the ${ }^{10} \mathrm{CH}_{2}-{ }^{12} \mathrm{CH}-{ }^{11} \mathrm{CH}_{2}-$ fragment in Fig. 1, if one assumes the interaction mechanism of Fig. 5. It is probable that the band at $1392 \mathrm{~cm}^{-1}$ could correspond to $\mathrm{C}-\mathrm{H}$ bending of the asymmetric ${ }^{12} \mathrm{C}$ located in the head of the side chain (Fig. 1). ${ }^{22}$

According to the SERS selection rules, the higher enhancement observed in the SERS spectrum will be that of vibrational modes developing through a direction perpendicular to the plane of the metal surface. Thus, the flat orientation of TOB moieties on the metal surface ac-

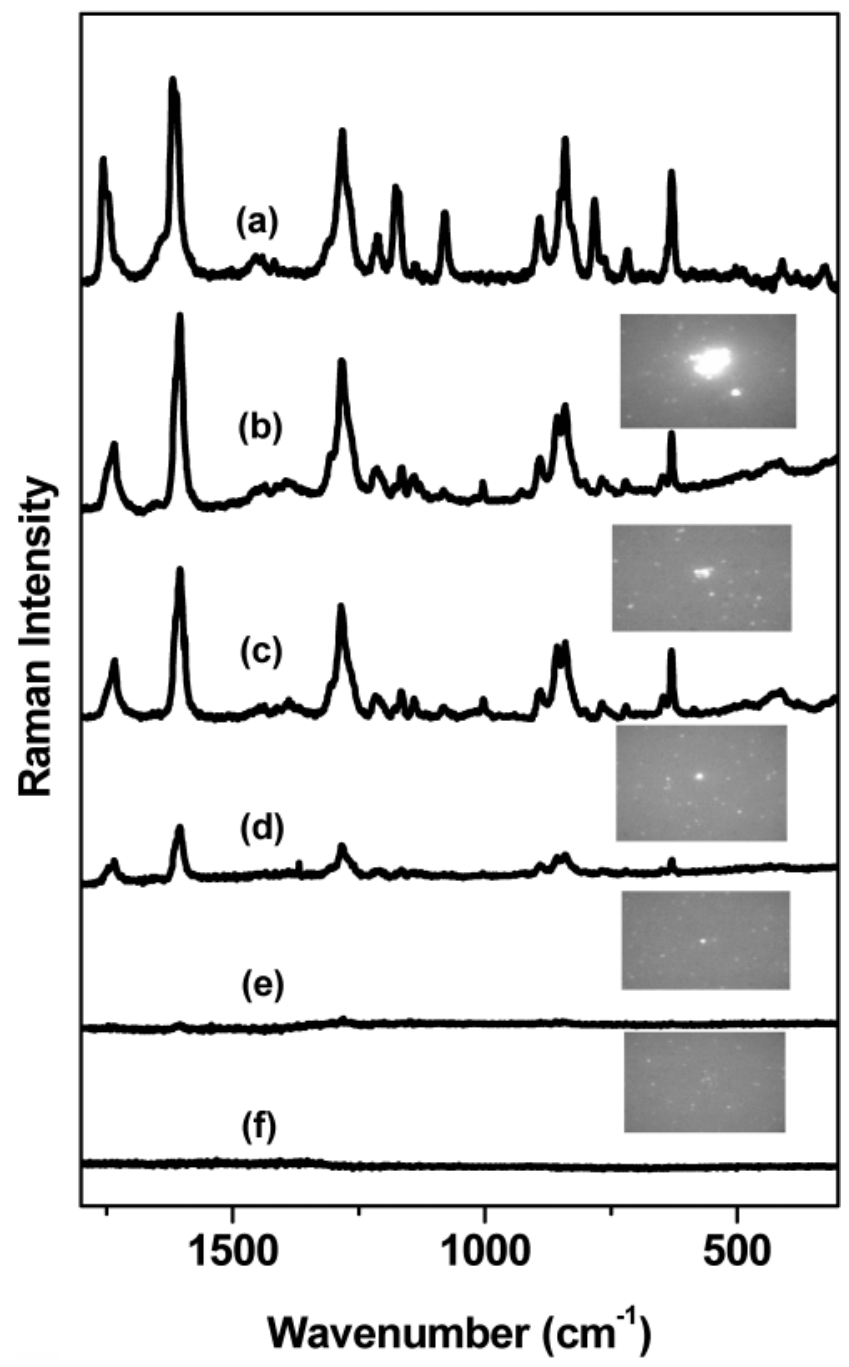

FIG. 6. Micro-Raman of PTOBDME in solid state $(\boldsymbol{a})$ and micro-SERS spectra of the polymer on the immobilized Ag nanoaggregates (prepared according to the method described in the Experimental section) shown in the direct optical images inserted on each spectrum, which have a different number $(n)$ of nanoparticles: $(\boldsymbol{b}) n>100 ;(\boldsymbol{c}) 25-30 ;(\boldsymbol{d}) 10-$ $12 ;(\boldsymbol{e})$ 4-6; $(\boldsymbol{f})$ non-aggregated particles. In all cases the excitation line was $785 \mathrm{~nm}$.

counts for the observed decrease of the ester vibrational modes such as the $\mathrm{C}=\mathrm{O}\left(\right.$ at $1733 \mathrm{~cm}^{-1}$ ) and $\mathrm{C}-\mathrm{O}$ stretching (at 1285 and $1223 \mathrm{~cm}^{-1}$ ) and the $\mathrm{C}=\mathrm{O}$ in-plane deformation at $630 \mathrm{~cm}^{-1}$, since these vibrations develop parallel to the metal surface. The same can be said for the in-plane ring modes such as the in-plane $\mathrm{C}-\mathrm{H}$ deformation appearing at $1200-1000 \mathrm{~cm}^{-1}$ and the ring breathing mode appearing at $840 \mathrm{~cm}^{-1}$ in p-substituted benzene rings. ${ }^{23}$ The $\mathrm{C}=\mathrm{C}$ stretching mode of the aromatic rings at $1606 \mathrm{~cm}^{-1}$ is still intense, although it is an in-plane vibration. The reason for this can be: (1) the high out-ofplane component predicted for this kind of vibration, taking into account that it involves the $\pi$-electrons of the aromatic moiety; or (2) an electronic resonance effect associated with a charge transfer between the adsorbate and the metal, which is one of the mechanisms implied in the overall SERS effect. The last assumption is supported by the fact that the $1733 / 1606 \mathrm{~cm}^{-1}$ ratio decreases in going from the Raman spectrum (Fig. 4a) to SERS at $1064 \mathrm{~nm}$ 

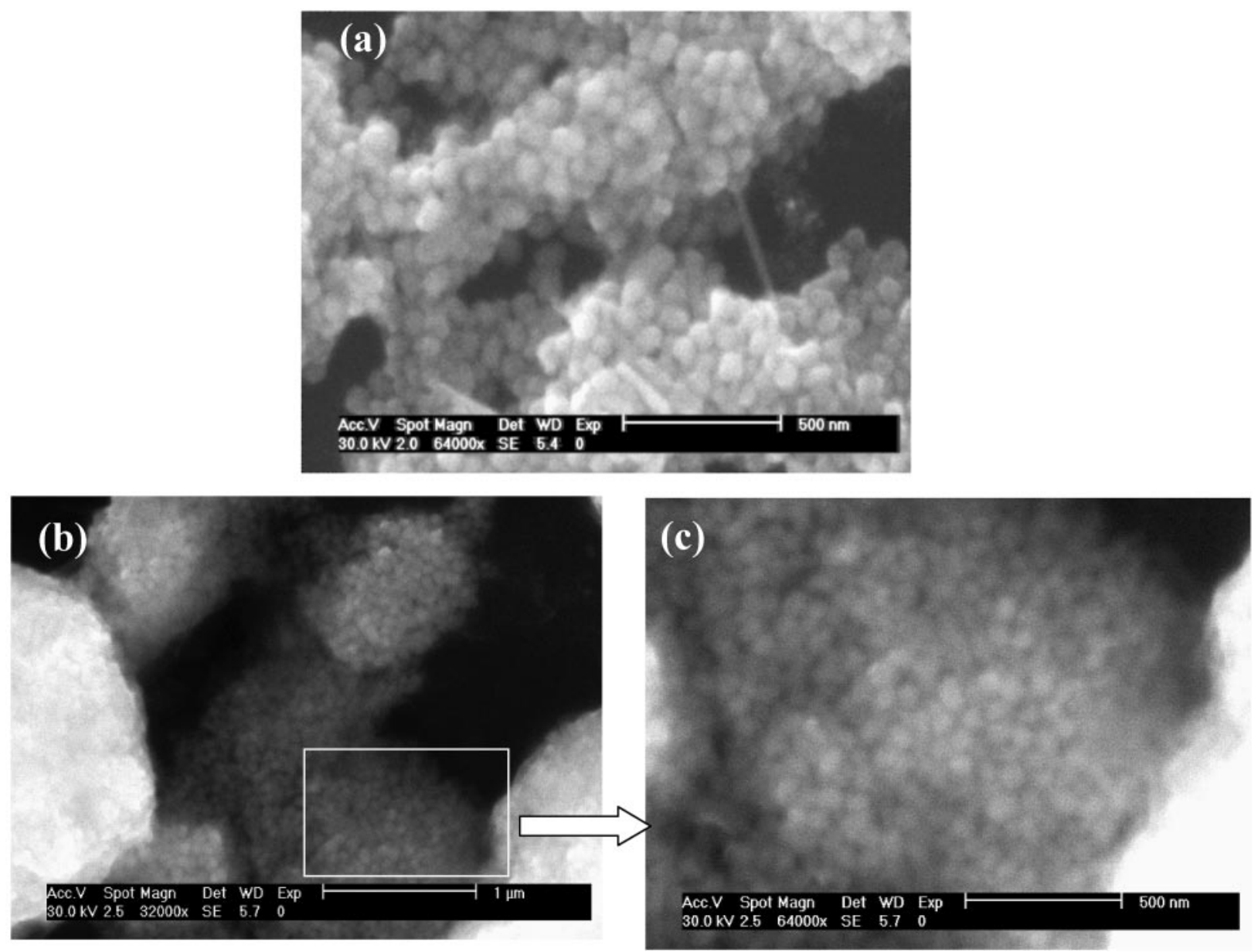

FIG. 7. ESEM micrographs of (a) massive citrate $\mathrm{Ag}$ nanoparticles and (b and $\mathbf{c}$ ) the same surfaces after adding PTOBDME suspended in $\mathrm{CHCl}_{3}$. Micrograph (c) is an amplification of the selected area shown in micrograph (b).

laser frequency (Fig. 4b) and to $514.5 \mathrm{~nm}$ frequency (Fig. $4 \mathrm{c}$ ), as corresponds to a higher resonance in the last case.

The SERS spectra also show an enhancement of bands between 1000 and $850 \mathrm{~cm}^{-1}$ (at 980, 930, 892, and 861 $\mathrm{cm}^{-1}$ ), which may include large contributions from outof-plane vibrations of the aromatic rings whose intensity is increased due to the predominant flat orientation of TOB triads, as stated by the SERS selection rules.

The adsorption of PTOBDME on immobilized Ag nanoparticles was also analyzed by micro-SERS. Figure 6 displays the micro-SERS spectra of the polymer compared to the micro Raman of the solid (Fig. 6a). The solid spectrum displays a large fluorescence background, which was corrected in the Fig. 6a spectrum. This fluorescence is markedly quenched upon polymer adsorption on the surface. The SERS spectra shown in Figs. 6b-6e correspond to the different $\mathrm{Ag}$ aggregates of the micrographs shown in the same figure. As can be seen, the intensity of the signal increases as the size of the aggregate increases, as predicted by the electromagnetic mechanism of the SERS intensification. ${ }^{11 a}$ The observation of any SERS signal depends on the aggregation of the nanoparticles. For non-aggregated particles (Fig. 6f) a very weak signal is registered. It is of note that the SERS signal is markedly enhanced when passing from 4-6 (Fig. 6e) to 25-30 nanoparticle aggregates (Fig. 6c). Nevertheless, from the latter aggregates to much larger ones (more than 100 particles, Fig. 6b) the intensity did not change very much. This is due to the fact that the number of active sites analyzed in the aggregates of Figs. 6c and $6 \mathrm{~b}$ is approximately the same. From the analysis of the SERS spectra, in comparison to the Raman of the solid, the tendency of the polymer to be adsorbed on the Ag immobilized nanoparticles under the uncoiled form is obvious, as was also deduced from the macro-Raman spectra. On the other hand, the spectral profile did not change significantly, which means that the polymer is uniformly adsorbed onto the Ag surface of the analyzed nanoparticles.

We have also analyzed the ESEM of massive Ag immobilized nanoparticles before and after adding PTOBDME. The ESEM micrographs of nanoparticle films prepared by immobilization of the Ag citrate colloid are shown in Fig. 7a, while Figs. $7 \mathrm{~b}$ and $7 \mathrm{c}$ display the $\mathrm{Ag}$ nanoparticles covered by PTOBDME at two different amplifications. A slight enlargement of the original particles is observed upon addition of the polymer on the metal spheres. The adsorption of PTOBDME on the Ag nanoparticles is very uniform. The nanoparticles seem to be uniformly covered by the polymer. Furthermore, the metal nanoparticles seem to be included in a polymer matrix and more separated from one another.

In Fig. 8 the spectral region corresponding to $\mathrm{C}-\mathrm{H}$ stretching vibrations is shown in more detail for both 


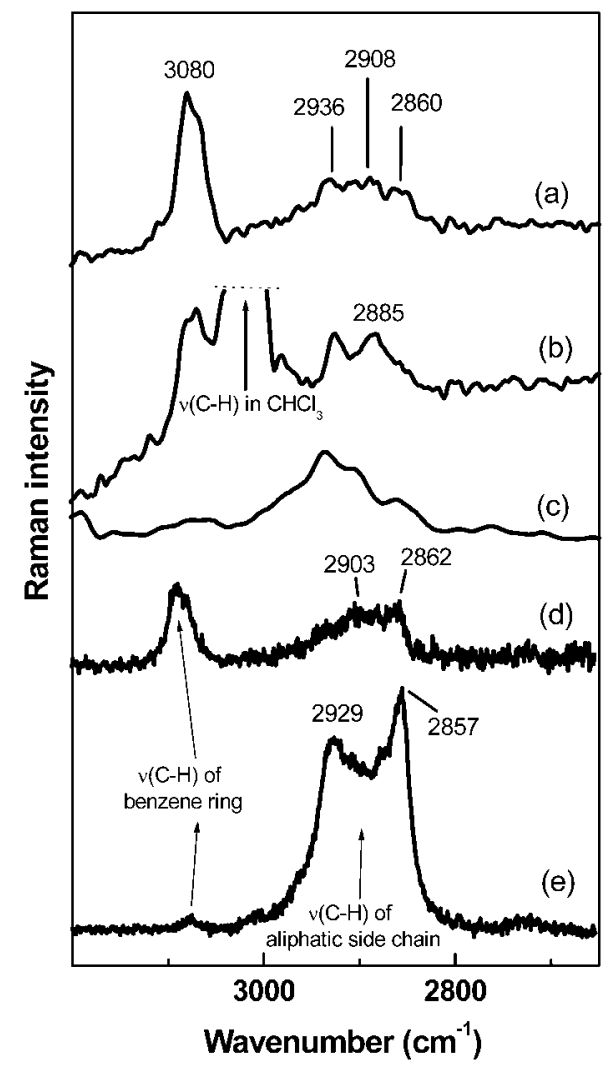

FIG. 8. Raman of PTOBDME (C-H stretching region) in (a) water suspension, $(\boldsymbol{b})$ in $\mathrm{CHCl}_{3}$, and (c) SERS spectrum on the colloid, all excited at $1064 \mathrm{~nm}$. Raman of PTOBDME in $(\boldsymbol{d})$ the solid state and $(\boldsymbol{e})$ SERS on immobilized Ag nanoparticles prepared according to the method described in the Experimental section, both obtained by excitation at $785 \mathrm{~nm}$.

macro- and micro-SERS spectra. Raman spectra of PTOBDME dispersed in water (Fig. 8a) and in $\mathrm{CHCl}_{3}$ (Fig. $8 b)$ are compared to SERS spectra of the polymer with laser excitation at $1064 \mathrm{~nm}$ (Fig. 8c) and $785 \mathrm{~nm}$ (Fig. $8 \mathrm{e})$, respectively. Figure 8d displays the micro-Raman of the solid. The $\mathrm{C}-\mathrm{H}$ stretching region is important from the point of view of the orientation investigation, as the aromatic $\mathrm{C}-\mathrm{H}$ stretching modes have a high in-plane component. ${ }^{24}$ In the normal Raman spectra (Figs. 8a, 8b, and $8 \mathrm{~d}$ ) the aromatic $\mathrm{C}-\mathrm{H}$ stretching bands, appearing at 3085-3070 $\mathrm{cm}^{-1}$, are much more intense than those of aliphatic groups $\left(2930-2850 \mathrm{~cm}^{-1}\right)$. On the contrary, in the SERS spectra (Figs. 8c and 8e) the relative intensity of these bands is inverted: the aromatic bands markedly decrease in relation to the aliphatic ones, becoming 20fold lower than the same band in aqueous suspension. This fact supports again a predominant parallel orientation of aromatic rings in relation to the surface, according to the SERS selection rules.

Furthermore, the aliphatic side chain conformation seems to be dramatically changed upon adsorption of the polymer on the surface, as indicated by the aliphatic $\mathrm{C}-\mathrm{H}$ stretching vibrations appearing below $3000 \mathrm{~cm}^{-1}$. In fact, the weakening of the $v_{\text {as }}\left(\mathrm{CH}_{2}\right)$ mode appearing at 2885 $\mathrm{cm}^{-1}$ in the $\mathrm{CHCl}_{3}$ (Fig. 8b), which is related to the lateral order of aliphatic chains, ${ }^{25}$ together with the relative increase of the $v_{\mathrm{s}}\left(\mathrm{CH}_{2}\right)$ mode at $2857-63 \mathrm{~cm}^{-1}$ in both SERS spectra (Figs. 8c and 8e), suggests a marked in- crease of the disorder structure of the polymer side chain when adsorbed on the metal surface. In fact, the spectrum of Fig. 8e displays a spectral profile that is very similar to that obtained for aliphatic chains at high temperature. ${ }^{26}$ In addition, the SERS registered at $1064 \mathrm{~nm}$ (Fig. 8c) displays a different spectral pattern in this region, with a prominent band at $2936 \mathrm{~cm}^{-1}$, which can have contributions from the residual citrate and its oxidation products still present on the metal surface and which are resonantly enhanced at $1064 \mathrm{~nm} .{ }^{26}$ This band did not appear in the micro-SERS of the polymer obtained on the immobilized aggregates due to the fact that the nanoparticles were thoroughly washed with water and, after, with ethanol in order to remove any contaminant compound existing in the Ag original colloids.

\section{CONCLUSION}

The thermotropic cholesteric liquid crystal polyester PTOBDME shows a high affinity to be adsorbed and selfassembled on Ag surfaces despite its very low solubility in aqueous media. The adsorption of the polymer involves a deep conformational change of the main chain, consisting in an opening of the helicoidal structure, adopted by PTOBDME in suspension, to an extended lineal polymer structure when the polymer is adsorbed on the metal surface. This structural change may be favored by an interaction of the metal with the aromatic rings of TOB triad via $\pi$-electronic interactions, leading to small torsional angles between them. Anther important structural consequence of this kind of interaction is the order decrease of the aliphatic side chain.

A previous Raman study of the conformation of PTOBDME in the solid state and suspended in different solvents helped us to attribute the marker bands related to the conformation of polymer chains. Namely, we have found important changes of the $v(\mathrm{C}=\mathrm{O}), v(\mathrm{C}=\mathrm{C})$, and $v(\mathrm{C}-\mathrm{O})$ modes, which can be related to the torsion of aromatic and ester planes around the direction of mesogens in the main chain. In addition $v(\mathrm{C}-\mathrm{H})$, motions can be related to the conformation of the aliphatic side chain. The attribution of all these bands was very important for assessing the interaction and orientation of PTOBDME on the used metal surfaces.

The micro Raman experiments demonstrated that the adsorption of the polymer on $\mathrm{Ag}$ nanoparticles is very homogeneous. The aggregation of particles is necessary for the observation of SERS. In micro-SERS an optimal SERS intensity was measured for aggregates having 20/25 nanoparticles, which corresponds to about $1 \mathrm{pg}$ of the polymer.

Raman spectroscopy was thus very helpful in the structural characterization of polymers in the free state or adsorbed on surfaces. The interaction of polymers like PTOBDME with metals could, then, be interesting in the preparation of functionalized surfaces, providing them with specific properties resulting from the joint combination of the metal and a polymer, with interesting applications in recognition phenomena. We are currently investigating these possible applications with PTOBDME.

\section{ACKNOWLEDGMENTS}

This work has been supported by Dirección General de Enseñanza Superior e Investigación Científica Project number BFM2001-2265 and 
Comunidad Autonoma de Madrid project number 07G/0042/2003. The authors acknowledge Dr. J. D. Gómez Varga for the ESEM measurements at the Institute of Polymer Science and Technology (CSIC).

1. H. Ringsdorf, B. Schlarb, and J. Venzmer, Angew. Chem., Int. Ed. Engl. 27, 113 (1988).

2. H. L. De Vries, Acta Crystallogr. 4, 219 (1951).

3. (a) S. E. Shields, Opt. Photon. News 3, 44 (1992); (b) D. R. Wiff and R. J. Reinert, Polymer 39, 5069 (1998); (c) A. Arias, T. Maillou, I. Moggio, D. Guillon, J. Le Moigne, and B. Geffroy, Synth. Metals 127, 229 (2002); (d) M. V. Kozlovsky, Synth. Metals 127, 67 (2002).

4. M. Pérez-Méndez and C. Marco, Acta Polym. 48, 502 (1997).

5. J. Fayos, S. Sanchez-Cortes, C. Marco, and M. Pérez-Méndez, J. Macromol. Sci. B: Phys. 40, 553 (2001).

6. M. Pérez-Méndez, J. Fayos, and C. R. Mateo, "Self-assembly of Cholesteric Liquid Crystal Polyesters and Their Stereoselective Interactions with Liposomes of DMPC", in Advances in Biochirality, G. Pályi, C. Zucchi, and L. Caglioti, Eds. (Elsevier Science S. A., Amsterdam, 1999), Chap. 24.

7. (a) F. Benmouna, B. Peng, J. Gapinski, A. Patkowski, J. Rühe, and D. Johannsmann, Liq. Cryst. 28, 1353 (2001); (b) D. H. Jung, I.-J. Park, Y. K. Choi, S. B. Lee, H. S. Park, and J. Rühe, Langmuir 18, 6133 (2002).

8. T. Fukazawa, T. Tadokoro, H. Toriumi, T. Akahane, and M. Kimura, Thin Solid Films 799, 313 (1998).

9. W. Hill, "Surface-enhanced Raman Scattering (SERS)", in Surface and Thin Film Analysis, H. Bubert and H. Jenett, Eds. (Wiley-VCH, Weinheim, 2002), Chap. 4, p. 254.

10. M. Fleischmann, P. J. Hendra, and A. J. McQuillan, Chem. Phys. Lett. 26, 163 (1974).

11. (a) M. Moskovits, Rev. Mod. Phys. 57, 783 (1985); (b) S. L. McCall and P. M. Platzman, Phys. Lett. A 77, 381 (1980).

12. (a) E. Burstein, Y. J. Chen, C. Y. Chen, S. Lundquist, and E. Tosatti, Solid State Comm. 29, 567 (1979); (b) A. Otto and I. Mrozek, J. Phys. Condens. Matter 4, 1143 (1992).
13. J. A. Creighton, "Selection Rules for Surface-Enhanced Raman Spectroscopy", in Spectroscopy of Surfaces, R. J. H. Clark and R. E. Hester, Eds. (John Wiley and Sons, Chichester, 1988), Chap. 2, p. 37.

14. P. C. Lee and D. Meisel, J. Phys. Chem. 86, 3991 (1982).

15. S. Sanchez-Cortes, J. V. García-Ramos, and G. Morcillo, J. Colloid Interface Sci. 167, 428 (1994)

16. S. Sanchez-Cortes, J. V. García-Ramos, G. Morcillo, and A. Tinti, J. Colloid Interface Sci. 175, 358 (1995)

17. (a) F. Boerio, S. K. Bahl, and G. E. McGraw, J. Polym. Sci. Polym Phys. Ed. 14, 1029 (1976); (b) I. M. Ward and M. A. Wilding, Polymer 18, 327 (1977); (c) G. D. McNally, N. J. Everall, J. M. Chalmers, and W. E. Smith, Appl. Spectrosc. 57, 44 (2003).

18. (a) E. Benedetti, F. Galleschi, E. Chiellini, and G. Galli, J. Polym Sci. B.: Phys. 27, 25 (1989); (b) D. Jehnichen, J. Tobisch, P. Friedel, and D. Pospiech, Polymer 37, 1463 (1996).

19. (a) G. Varsányi, Assignments for Vibrational Spectra of Seven Hundred Benzene Derivatives (Adam Hilger, London, 1974); (b) M. C. Alvarez-Ros, S. Sanchez-Cortes, and J. V. Garcia-Ramos, Spectrochim. Acta, Part A 56, 2471 (2000).

20. M. Pérez-Méndez, R. Marsal-Berenguel, L. Garrido, and M. Martín-Pastor, Macromolecules 36, 8049 (2003).

21. (a) T. T. Wong, J. Siminovitch, and H. H. Mantsch, Biochim. Biophys. Acta 947, 139 (1988); (b) A. T. Tu, Raman Spectroscopy in Biology: Principles and Applications (John Wiley and Sons, New York, 1982).

22. N. B. Colthup, L. H. Daly, and S. E. Wiberly, Introduction to Infrared and Raman Spectroscopy (Academic Press, New York, 1975), 2nd ed.

23. S. Sanchez-Cortes and J. V. Garcia-Ramos, J. Colloid. Interface Sci. 231, $98(2000)$.

24. M. Moskovits and J. S. Suh, J. Phys. Chem. 92, 6327 (1988).

25. G. Zerbi and M. Del Zoppo, "Vibrational Spectroscopy as a Probe of Structural Order/Disorder in Chain Molecules and Polymers", in Modern Polymer Spectroscopy, G. Zerbi, Ed. (Wiley-VCH, New York, 1999), Chap. 3, p. 87.

26. J. V. García-Ramos and S. Sanchez-Cortes, J. Raman Spectroc. 29 , 365 (1998) 